\title{
Papers
}

\section{Recurrence of pre-eclampsia across generations: exploring fetal and maternal genetic components in a population based cohort}

\author{
Rolv Skjærven, Lars J Vatten, Allen J Wilcox, Thorbjørn Rønning, Lorentz M Irgens, Rolv Terje Lie
}

\begin{abstract}
Objectives To assess the impact on risk of pre-eclampsia of genes that work through the mother, and genes of paternal origin that work through the fetus.

Design Population based cohort study.

Setting Registry data from Norway.

Participants Linked generational data from the medical birth registry of Norway (1967-2003): 438597 mother-offspring units and 286945 father-offspring units.

Main outcome measures Pre-eclampsia in the second generation.

Results The daughters of women who had pre-eclampsia during pregnancy had more than twice the risk of pre-eclampsia themselves (odds ratio 2.2, 95\% confidence interval 2.0 to 2.4) compared with other women. Men born after a pregnancy complicated by pre-eclampsia had a moderately increased risk of fathering a pre-eclamptic pregnancy $(1.5,1.3$ to 1.7$)$. Sisters of affected men or women, who were themselves born after pregnancies not complicated by pre-eclampsia, also had an increased risk (2.0, 1.7 to 2.3). Women and men born after pre-eclamptic pregnancies were more likely to trigger severe pre-eclampsia in their own (or their partner's) pregnancy (3.0, 2.4 to 3.7, for mothers and 1.9, 1.4 to 2.5 , for fathers).

Conclusions Maternal genes and fetal genes from either the mother or father may trigger pre-eclampsia. The maternal association is stronger than the fetal association. The familial association predicts more severe pre-eclampsia.
\end{abstract}

\section{Introduction}

The heritable aspects of pre-eclampsia, which occurs in 3-5\% of pregnancies, are complex. ${ }^{1}$ The first pattern to be identified was the tendency for the risk of pre-eclampsia to be passed from mother to daughter. Specifically, women who were born after a pregnancy affected by pre-eclampsia are themselves at increased risk of pre-eclampsia in their own pregnancies. ${ }^{2-4}$ This risk could reflect at least two genetic pathways: transmission to the daughter of genes that enhance maternal susceptibility to preeclampsia, or transmission from the daughter to her fetus of fetal genes that are capable of triggering pre-eclampsia.

Recent studies have shown that an increased risk of pre-eclampsia could also be transmitted through the father. One study showed that men who had fathered a pre-eclamptic pregnancy in one woman had an increased risk of fathering a pre-eclamptic pregnancy in another woman. ${ }^{5}$ In another study, men who themselves were born after a pre-eclamptic pregnancy conveyed an increased risk of pre-eclampsia in pregnancies they subsequently fathered. ${ }^{6}$ These findings support the hypothesis that the father's genes can be passed to the fetus and increase the risk of pre-eclampsia.

We explored these two pathways of genetic transmission of risk of pre-eclampsia using linked birth data between family members as recorded in the medical birth registry of Norway.

\section{Methods}

Population based generational data

We used data from the medical birth registry of Norway, a population based registry of all births in Norway since 1967 (more than 2.2 million births). ${ }^{7}$ Up to 2003,238617 women born in Norway in 1967 or later (and therefore registered in the medical birth registry) had given birth to 438597 singleton infants. Similarly, 158340 men born in 1967 or later had fathered 286945 singleton infants. The number of mothers is considerably higher because mothers are generally younger than fathers and $4.4 \%$ of fathers were unknown and could not be identified. Fifty eight per cent of the mothers were born during 1967-71 and 89\% during 1967-76. For the fathers the figures were $65 \%$ and $94 \%$, respectively.

We used these data to study whether women and men who themselves were born after a pregnancy complicated by pre-eclampsia have a higher risk of parenting a pre-eclamptic pregnancy, compared with those who had no family history of pre-eclampsia. We estimated risk related to their first pregnancy or to the second pregnancy if pre-eclampsia had not occurred in the first pregnancy.

We also assessed the risk of pre-eclampsia in any siblings who were born after an unaffected pregnancy to study whether such offspring with a family history of pre-eclampsia could none the less pass on an increased risk to the next generation. We identified families with at least two siblings (male or female) born since 1967 who later became parents. We identified 105525 pairs of siblings ( $45.6 \%$ of these were first and second births), and then excluded the 185 pairs where both siblings from the first generation were born after a pregnancy complicated by pre-eclampsia.

\section{Definition of pre-eclampsia}

We included pregnancies with a specified diagnosis of pre-eclampsia and pregnancies with a combination of pregnancy induced hypertension and proteinuria. Obstetric guidelines in Norway define pre-eclampsia as an increase in blood pressure to at least $140 / 90 \mathrm{~mm} \mathrm{Hg}$ after the 20th week of gestation. ${ }^{8}$ Compared with measures before the 20th week, either the diastolic blood pressure has to be at least $15 \mathrm{~mm} \mathrm{Hg}$ higher or the systolic blood pressure has to be at least $30 \mathrm{~mm} \mathrm{Hg}$ higher. Proteinuria (protein excretion at least $0.3 \mathrm{~g}$ per 24 hours) also 
Table 1 Risk of pregnancy* affected by pre-eclampsia in women and men† who themselves were born after a pregnancy complicated by pre-eclampsia, Norway, 1967-2003

\begin{tabular}{|c|c|c|c|c|}
\hline & Birth order & No of offspring & No $(\%)$ of affected pregnancies & Odds ratio $(95 \% \mathrm{CI}) \neq$ \\
\hline \multicolumn{5}{|c|}{ Women } \\
\hline \multicolumn{5}{|c|}{ Maternal pre-eclampsia: } \\
\hline Yes & First & 2768 & $299(10.8)$ & $2.2(1.9$ to 2.5$)$ \\
\hline No & First & 91338 & $4794(5.2)$ & $1.0 \S$ \\
\hline Yes & Subsequent & 1977 & $200(10.1)$ & $2.1(1.8$ to 2.4$)$ \\
\hline No & Subsequent & 142288 & $7261(5.1)$ & $1.0 \S$ \\
\hline \multicolumn{5}{|l|}{ Men } \\
\hline \multicolumn{5}{|c|}{ Maternal pre-eclampsia: } \\
\hline Yes & First & 1908 & $146(7.7)$ & $1.4(1.2$ to 1.7$)$ \\
\hline No & First & 60137 & $3274(5.4)$ & $1.0 \S$ \\
\hline Yes & Subsequent & 1270 & $101(8.0)$ & $1.5(1.2$ to 1.8$)$ \\
\hline No & Subsequent & 94917 & $5154(5.4)$ & $1.0 \S$ \\
\hline
\end{tabular}

Confined to first pregnancies of women and men.

tFor men this is No (\%) in their partners.

łPooled odds ratios (Mantel-Haenszel): 2.2 (2.0 to 2.4) for women and 1.5 (1.3 to 1.7) for men. §Reference category.

has to be present. We further divided pre-eclampsia according to gestational age at delivery, as either term (37-42 weeks) or preterm (before 37 weeks) pre-eclampsia.

Since 1999, clinical severity, divided into mild or severe and according to whether clinical signs were evident early $(<34$ weeks) in pregnancy, has been added to the registration of pre-eclampsia. This information allowed us to explore the main results in greater detail in a subgroup of the population.

\section{Statistical analysis}

For women born after a pre-eclamptic pregnancy, we estimated their own risk of pre-eclampsia in subsequent pregnancies compared with the risk in women who were not born after a pregnancy complicated by pre-eclampsia. We compared the risk of fathering a pre-eclamptic pregnancy in men who were born after a pre-eclamptic pregnancy and men who were not. For sisters and brothers who were born after another pregnancy without pre-eclampsia, we also estimated the risk of pre-eclampsia compared with the risk in women and men with no family history.

We used SPSS for Windows (release 12.0), Stata (version 8), and StatXact (version 6), with logistic regression and multinomial logistic regression analysis to estimate odds ratios and $95 \%$ confidence intervals.

\section{Results}

\section{Pre-eclampsia related to first and second birth}

Women who were born after a pre-eclamptic pregnancy had more than twice the risk of having pre-eclampsia in their first pregnancy compared with other women (odds ratio 2.2, 95\% confidence interval 2.0 to 2.4, table 1). For men who were born after a pregnancy complicated by pre-eclampsia, the risk of preeclampsia in the first pregnancy of their partner was moderately increased compared with men who were born after a pregnancy not complicated by pre-eclampsia (1.5, 1.3 to 1.7 , table 1$)$. For both men and women, these associations were remarkably stable, regardless of their own birth order.

We also estimated the familial risk of pre-eclampsia related to the second birth, provided that the first pregnancy was not affected by pre-eclampsia. Although the baseline risk of pre-eclampsia for the second birth was lower than for the first $(1.5 \%)$, we found that women who themselves were born after a pre-eclamptic pregnancy still had more than twice the risk (2.3, 1.8 to 2.9 ) of pre-eclampsia related to their second birth compared with other women (data not shown). The results were similar for men (1.5, 1.1 to 2.1). The combination of both parents having been born after an affected pregnancy did not occur more often than expected so simultaneous estimation, with logistic regression, gave similar odds ratio estimates: 2.1 (2.0 to 2.4 ) and 1.5 (1.3 to 1.7$)$, respectively.

\section{Pre-eclampsia in brothers and sisters}

Sisters who were not themselves born after a pregnancy complicated by pre-eclampsia were none the less at increased risk of pre-eclampsia compared with women with no family history of pre-eclampsia $(2.0,1.7$ to 2.3 , table 2). For brothers not themselves born after a pre-eclamptic pregnancy, the risk of fathering a pre-eclamptic pregnancy was similar to that in men with no family history (1.1, 0.9 to 1.4$)$. Birth order did not influence these results. The figure illustrates the results shown in tables 1 and 2.

Table 2 Risk of pregnancy* affected by pre-eclampsia in sisters and brothers $\dagger$ who were born after a pregnancy not complicated by pre-eclampsia, Norway, 1967-2003

\begin{tabular}{ccc} 
No of siblings & $\begin{array}{c}\text { No }(\%) \text { with } \\
\text { pre-eclampsia }\end{array} \quad$ Odds ratio $(95 \% \mathrm{Cl}) \ddagger$ \\
\hline
\end{tabular}

\begin{tabular}{|c|c|c|c|}
\hline$\overline{\text { Younge }}$ & & & \\
\hline Mother & sia in firs & & \\
\hline Yes & 1146 & $117(10.2)$ & 2.1 (1.7 to 2.5$)$ \\
\hline No & 63736 & $3327 \quad(5.2)$ & $1.0 \S$ \\
\hline Younge & & & \\
\hline Mother & sia in firs & & \\
\hline Yes & 728 & $48 \quad(6.6)$ & 1.2 (0.9 to 1.5$)$ \\
\hline No & 38754 & $2251(5.8)$ & $1.0 \S$ \\
\hline Older s & & & \\
\hline Mother & sia in sec & & \\
\hline Yes & 580 & $54(9.3)$ & 1.9 (1.4 to 2.5$)$ \\
\hline No & 59205 & 3116 (5.3) & $1.0 \S$ \\
\hline Older & & & \\
\hline Mother & sia in sec & & \\
\hline Yes & 396 & $23(5.8)$ & $1.0(0.7$ to 1.6$)$ \\
\hline No & 43285 & $2461 \quad(5.7)$ & $1.0 \S$ \\
\hline
\end{tabular}


Table 3 Severity of pre-eclampsia in women and in men's partners, by maternal history of pre-eclampsia, Norway, 1999-2003

\begin{tabular}{|c|c|c|c|}
\hline & $\begin{array}{c}\text { No of } \\
\text { pregnancies }\end{array}$ & $\begin{array}{c}\text { No }(\%) \text { of mothers with } \\
\text { pre-eclampsia* }\end{array}$ & $\begin{array}{c}\text { Odds ratio }(95 \% \\
\text { CI) } \dagger\end{array}$ \\
\hline \multicolumn{4}{|c|}{ Women's pregnancy } \\
\hline No pre-eclampsia & 131805 & $2357(1.8)$ & $1.0 \neq$ \\
\hline \multicolumn{4}{|c|}{ Type of pre-eclampsia: } \\
\hline Mild & 3839 & $136(3.5)$ & 2.1 (1.8 to 2.4$)$ \\
\hline Severe/early & 1792 & $90(5.0)$ & 3.0 (2.4 to 3.7$)$ \\
\hline \multicolumn{4}{|c|}{ Pregnancy in men's partners } \\
\hline No pre-eclampsia & 96405 & $1905(2.0)$ & $1.0 \ddagger$ \\
\hline \multicolumn{4}{|c|}{ Type of pre-eclampsia: } \\
\hline Mild & 3014 & $67(2.2)$ & 1.2 (1.0 to 1.5$)$ \\
\hline Severe/early & 1340 & $40(3.0)$ & 1.9 (1.4 to 2.5$)$ \\
\hline
\end{tabular}

*For pregnancy in men's partners, this was man's mother.

†Estimated with multinomial logistic regression.

‡Reference category.

\section{Clinical severity of pre-eclampsia}

In the subgroup of women who had given birth since 1999, those who had clinically severe or early onset pre-eclampsia were much more likely to have been born after a pre-eclamptic pregnancy themselves compared with women born after an unaffected pregnancy $(3.0,2.4$ to 3.7 , table 3). Men who had fathered a pregnancy with severe or early pre-eclampsia were also more likely to have been born after a pre-eclamptic pregnancy themselves compared with men who had not fathered an affected pregnancy $(1.9,1.4$ to 2.5$)$.

\section{Discussion}

Men born after a pre-eclamptic pregnancy are apparently more likely to carry fetal alleles capable of triggering pre-eclampsia. These men then pass the fetal risk alleles to their offspring, increasing their partner's risk of pre-eclampsia. The same presumably occurs for women born after pre-eclamptic pregnancies who can then pass susceptibility alleles to their own fetuses. In addition, women born after pre-eclamptic pregnancies may inherit from their mother genes that confer maternal susceptibility to pre-eclampsia. This maternal susceptibility can pass from mother to daughter but not from mother to son.

The purest expression of maternal genes would be among women not themselves born after a pre-eclamptic pregnancy but whose mothers had had pre-eclampsia in another pregnancy. Such women have twice the risk of pre-eclampsia in their own pregnancies compared with other women. The purest expression of fetal genes predisposing to pre-eclampsia would be among the offspring of men who had themselves triggered preeclampsia as fetuses. Such men have a 50\% increased risk of fathering a pre-eclamptic pregnancy. Women who were born after a pre-eclamptic pregnancy potentially carry both types of genetic predisposition-maternal and fetal-and seem to be at highest risk (figure).

\section{Comparisons with other studies}

Evidence for maternal predisposition to pre-eclampsia has been reported before. ${ }^{40-13}$ A population based case-control study from Utah, US, showed that women born after a pregnancy complicated by pre-eclampsia were more likely themselves to have pre-eclampsia. ${ }^{6}$

Another population based study in Norway supports the hypothesis that the father's genes also contribute to the risk of pre-eclampsia. ${ }^{5}$ In that study, men who fathered a pre-eclamptic pregnancy in one woman were also more likely to father a pre-eclamptic pregnancy in other women. The Utah study also supported a paternal predisposition, ${ }^{6}$ with men born of affected pregnancies at increased risk of fathering a pre-eclamptic pregnancy.

\section{Strength and weaknesses}

Among women and men who had a family history of pre-eclampsia, some were first born and others were born second or later. The familial association with pre-eclampsia was independent of birth order. As expected, the absolute risk of preeclampsia was reduced with increasing birth order, but the familial association was consistent, strengthening the evidence of an association.

Our cohort data are based on mandatory reporting in a population based registry over a 37 year period. The large study size and the standardised collection of data provide high precision in the estimates of effect. Moreover, the cohort design, comprising a whole population, reduces the possibility that selection bias can explain our findings. However, we cannot exclude potential confounding by other risk factors for pre-eclampsia. ${ }^{14}$ For example, obesity before pregnancy may be shared by mothers and daughters and may contribute to their shared risk of pre-eclampsia. In Norway, however, obesity of the degree that increases the risk of pre-eclampsia was relatively uncommon over the time of the study. ${ }^{15}$ Also, recent evidence suggests that the association with obesity may be limited to the relatively mild type of pre-eclampsia with term delivery. ${ }^{16} \mathrm{We}$ found a stronger familial association for clinically severe than for mild pre-eclampsia, which may decrease the likelihood that obesity could have confounded our results.

Other factors, such as interval between births, ${ }^{9}$ change of partner between births, ${ }^{9}{ }^{17}$ chronic hypertension, ${ }^{18}$ and smoking in pregnancy, ${ }^{19}$ have also been associated with the risk of pre-eclampsia. Although confounding by any one of these factors cannot be excluded, it seems unlikely that such factors could produce the specific contrasts within affected families that we see here. In the subgroup of women who had given birth since 1999, we had more detailed information on potentially 
confounding factors. In a supplementary analysis in this subgroup, we adjusted for length of gestation, diabetes in pregnancy, smoking early in pregnancy, and maternal age and education. This adjustment did not substantially influence the results.

\section{Maternal or fetal genes}

It seems reasonable to attribute the observed patterns of familial predisposition to genetic inheritance. Daughters born after a pre-eclamptic pregnancy may carry their mothers' susceptibility genes, as well as genes from either parent that operate through the fetus. ${ }^{156}$ Their sisters who were born after pregnancies not complicated by pre-eclampsia would be at lower risk as they are less likely to be carrying the genes that operate through the fetus. Still they are just as likely to be carrying their mothers' susceptibility genes. Thus, sisters of affected men and women have about twice the risk of women with no family history of pre-eclampsia.

For men who were born after an affected pregnancy, an increased risk of fathering a pre-eclamptic pregnancy can be attributed solely to paternal factors. Brothers who were born after a pregnancy without pre-eclampsia would have a low probability of carrying fetal risk genes, which is confirmed by the near baseline risk of pre-eclampsia among pregnancies they father.

\section{Mild or severe pre-eclampsia}

Recent epidemiological studies ${ }^{2021}$ have supported the hypothesis that pre-eclampsia comprises several pathogenetic entities, rather than a single process, with varying degrees of clinical severity. ${ }^{22}$ Placental dysfunction could cause pre-eclampsia in babies with low weight for gestational age ${ }^{122-24}$ but is less likely for larger babies. ${ }^{202}$ The familial association is stronger for preterm than for term pre-eclampsia. When we confined analysis to preterm pre-eclampsia in the first generation, the odds ratios were 2.6 (2.0 to 3.4) and 1.7 (1.2 to 2.5) for women and men, respectively. For preterm pre-eclampsia in the second generation the odds ratios were 2.8 (2.3 to 3.3 ) and 1.7 (1.3 to 2.2). This may suggest that genetic susceptibility to pre-eclampsia, either through maternal or paternal genes, is more likely to cause clinically severe disease..$^{25}$ If changes over time in reporting of pre-eclampsia include more mild pre-eclampsia, recurrence of pre-eclampsia between generations will be reduced. The proportion of preterm pre-eclampsia was $10 \%$ in the first period (parents) and 22\% in the last period (offspring).

\section{Conclusions}

Both maternal and paternal factors contribute to the risk of preeclampsia. The risk through affected mothers is higher, presumably because these mothers carry susceptibility genes and also transmit independent genetic risk factors to their fetus. The risk through affected fathers is lower because fathers transmit only fetal risk factors. Also, the differences that we observed between sisters and brothers suggest that genes that determine maternal susceptibility to pre-eclampsia differ from the paternal genes that may trigger pre-eclampsia through the fetus. Finally, familial associations are stronger for the clinically more severe types of pre-eclampsia.

Contributors: RS, LJV, and RTL planned the project, analysed the data, and drafted the manuscript. AJW and LMI advised on study design and analytic strategy and contributed to the writing of the paper. TR was responsible for data management and participated in the analysis of data. RS is guarantor. Funding: Norwegian Medical Research Council.

Competing interests: None declared.

Ethical approval: Medical Birth Registry of Norway.

1 Roberts JM, Cooper DW. Pathogenesis and genetics of pre-eclampsia. Lancet 2001;357:53-6.

\section{What is already known on this topic}

Maternal susceptibility to pre-eclampsia can be passed from mother to daughter

Less is known about fetal propensity to trigger pre-eclampsia and the transmission of such propensity through the father or mother

\section{What this study adds}

Men and women who were born after pre-eclamptic pregnancies contribute to increased risk of pre-eclampsia in the next generation

The risk through affected mothers is higher, presumably because these mothers carry susceptibility genes and also transmit independent genetic risk factors to their fetus

Risk through affected fathers is lower presumably because fathers transmit only fetal risk factors

Other familial patterns are consistent with independent genetic risk in the mother and the fetus and the associations seem to be stronger for the clinically more severe types of pre-eclampsia

2 Chesley LC, Cooper DW. Genetics of hypertension in pregnancy: possible single gene control of pre-eclampsia and eclampsia in the descendants of eclamptic women. BJOG 1986;93:898-908.

3 Cooper DW, Hill JA, Chesley LC, Bryans CI. Genetic control of susceptibility to eclampsia and miscarriage. BJOG 1988;95:644-53.

4 Arngrimsson R, Bjornsson S, Geirsson RT, Bjornsson H, Walker JJ, Snaedal G. Genetic and familial predisposition to eclampsia and pre-eclampsia in a defined population. BJOG 1990;97:762-9.

5 Lie RT, Rasmussen S, Brunborg H, Gjessing HK, Lie-Nielsen E, Irgens LM. Fetal and maternal contributions to risk of pre-eclampsia: population based study. BMJ 1998:316:1343-7.

6 Esplin MS, Fausett MB, Fraser A, Kerber R, Mineau G, Carrillo J, et al. Paternal and maternal components of the predisposition to preeclampsia. $N$ Engl $J$ Med 2001;344:867-72.

7 Irgens LM. The medical birth registry of Norway. Epidemiological research and surveillance throughout 30 years. Acta Obstet Gynecol Scand 2000;79:435-9.

8 Dalaker K, Berle EJ. Clinical guidelines in obstetrics 1999. The Norwegian Society of Obstetrics and Gynecology. Oslo: Norwegian Medical Association, 1999.

9 Skjaerven R, Wilcox AJ, Lie RT. The interval between pregnancies and the risk of preeclampsia. N Engl J Med 2002;346:33-8

10 Cincotta RB, Brennecke SP. Family history of pre-eclampsia as a predictor for pre-eclampsia in primigravidas. Int J Gynaecol Obstet 1998;60:23-7.

11 Mogren I, Hogberg U, Winkvist A, Stenlund H. Familial occurrence of preeclampsia. Epidemiology 1999;10:518-22.

12 Nilsson E, Salonen Ros H, Cnattingius S, Lichtenstein P. The importance of genetic and environmental effects for pre-eclampsia and gestational hypertension: a family study. BJOG 2004;111:200-6.

13 Pawitan Y, Reilly M, Nilsson E, Cnattingius S, Lichtenstein P. Estimation of genetic and environmental factors for binary traits using family data. Stat Med 2004;23:449-65.

14 Broughton Pipkin F. Risk factors for preeclampsia. N Engl J Med 2001;344:925-6.

15 Engeland A, Bjorge T, Tverdal A, Sogaard AJ. Obesity in adolescence and adulthood and the risk of adult mortality. Epidemiology 2004;15:79-85.

16 Odegard RA, Vatten LJ, Nilsen ST, Salvesen KA, Austgulen R. Risk factors and clinical manifestations of pre-eclampsia. BJOG 2000;107:1410-6

17 Dekker GA, Robillard PY, Hulsey TC. Immune maladaptation in the etiology of preeclampsia: a review of corroborative epidemiologic studies. Obstet Gynecol Surv 1998;53:377-82.

18 Sibai BM, Lindheimer M, Hauth J, Caritis S, Van Dorsten P, Klebanoff M, et al. Risk factors for preeclampsia, abruptio placentae, and adverse neonatal outcomes among women with chronic hypertension. National Institute of Child Health and Human Development Network of Maternal-Fetal Medicine Units. N Engl J Med 1998;339:66771.

19 Cnattingius S, Mills JL, Yuen J, Eriksson O, Salonen H. The paradoxical effect of smoking in preeclamptic pregnancies: smoking reduces the incidence but increases the rates of perinatal mortality, abruptio placentae, and intrauterine growth restriction. Am J Obstet Gynecol 1997;177:156-61.

20 Odegard RA, Vatten LJ, Nilsen ST, Salvesen KA, Austgulen R. Preeclampsia and fetal growth. Obstet Gynecol 2000;96:950-5.

21 Xiong X, Demianczuk NN, Saunders LD, Wang FL, Fraser WD. Impact of preeclampsia and gestational hypertension on birth weight by gestational age. Am J Epidemiol 2002;155:203-9.

22 Ness RB, Roberts JM. Heterogeneous causes constituting the single syndrome of preeclampsia: a hypothesis and its implications. Am J Obstet Gynecol 1996;175:1365-70.

23 Teasdale F. Histomorphometry of the human placenta in maternal preeclampsia. Am J Obstet Gynecol 1985;152:25-31. 
24 Ghidini A, Salafia CM, Pezzullo JC. Placental vascular lesions and likelihood of diagnosis of preeclampsia. Obstet Gynecol 1997;90:542-5.

25 Vatten LJ, Skjaerven R. Is pre-eclampsia more than one disease? BJOG 2004;11:298-302.

(Accepted 5 July 2005)

doi $10.1136 /$ bmj.38555.462685.8F

Section for Epidemiology and Medical Statistics, Department of Public Health and Primary Health Care, University of Bergen, Norway

Rolv Skjærven professor

Rolv Terje Lie professor

Norwegian University of Science and Technology, Faculty of Medicine, Trondheim,
Norway

Lars J Vatten professor

National Institute of Environmental Health Sciences, Research Triangle Park, NC, USA

Allen J Wilcox

Division of Genes and Environment, Norwegian Institute of Public Health, Oslo, Norway

Thorbjørn Rønning statistician

Medical Birth Registry of Norway, Norwegian Institute of Public Health, Bergen, Norway

Lorentz M Irgens professor

Correspondence to: R Skjærven Rolv.Skjaerven@smis.uib.no 